\title{
ISOMORPHISMS AND AUTOMORPHISMS OF UNIVERSAL HEFFALUMP LIE ALGEBRAS
}

\author{
S. BERMAN ${ }^{1}$
}

\begin{abstract}
The classification of a family of infinite dimensional Lie algebras is carried out, and a determination of their automorphism groups, in certain cases is supplied.
\end{abstract}

Introduction. The theory of finite dimensional simple Lie algebras over algebraically closed fields of characteristic zero achieves a classification of such algebras and a description of their automorphism groups (see [4]). To these algebras one attaches a finite Cartan matrix and the classification states that two such algebras are isomorphic if and only if their Cartan matrices are equivalent [see $\$ 1$ for definitions] and that there are only nine types of equivalence classes of finite Cartan matrices. The known proofs of this require detailed knowledge of the automorphism groups, which in turn leads to conjugacy of the Cartan subalgebras, and make heavy use of the finite dimensionality of the algebras.

In $\$ 1$ we will describe the class of Heffalump Lie algebras which generalize and include the class of algebras mentioned above. The name Heffalump was introduced in [10] as a generalization of the terminology introduced by Freudenthal and de Vries in their book "Linear Lie Groups" where the split 3 dimensional simple Lie algebra is said to be generated by a Hef triple. Our major concern in this paper is with the universal Heffalump algebras, and we completely solve the classification problem for these algebras. This leads to the determination of their automorphism groups when the Cartan matrix defining the algebras is not of Euclidean type and the base field has characteristic zero. The algebras we consider are infinite dimensional and have large subalgebras which are free Lie algebras. This fact enables us to supply a version of conjugacy, Theorem 3 below, which in turn leads easily to the classification, which is dealt with in $\$ 2$.

It is perhaps worthwhile to mention that the classification of the reduced Heffalump algebras, and the determination of their automorphism groups, is a major unsolved problem and appears much more difficult than the one treated here. Moody and Teo, in [9], discuss the group of invariant automor-

Received by the editors March 10, 1976.

AMS (MOS) subject classifications (1970). Primary 17B65, 17B05, 17B40.

${ }^{1}$ The author gratefully acknowledges the support of a National Research Council of Canada grant while this work was being done. 
phisms and show it is a group with BN pair. However, the relationship of this group to the full automorphism group is not known, because there is no version of conjugacy for these algebras. Also, in [3], it is shown that the nullity of the Cartan matrix defining the reduced Heffalump algebra is an invariant of the algebra, and that there are an infinite number of isomorphism classes of these algebras, but a complete set of invariants is unknown.

1. Description of the algebras and a version of conjugacy. An $l \times l$ matrix $\left(A_{i j}\right)$ with integer entries is a Cartan matrix if $A_{i i}=2, A_{i j}=0$ if and only if $A_{j i}=0, A_{i j} \leqslant 0$ if $i \neq j$, for $1 \leqslant i, j \leqslant l$. These generalize the well-known nine types of finite Cartan matrices which arise in the classification of the classical simple Lie algebras. Associated to each Cartan matrix is its CoxeterDynkin diagram, and we say the matrix is indecomposable if its diagram is connected. Two Cartan matrices, $\left(A_{i j}\right)$ and $\left(B_{i j}\right)$, of size $l \times l$ and $l^{\prime} \times l^{\prime}$ respectively, are equivalent if and only if $l=l^{\prime}$ and there is a permutation $\pi$ of $\{1, \ldots, l\}$ such that $A_{\pi(i), \pi(j)}=B_{i j}$ for $1 \leqslant i, j \leqslant l$. Throughout, we only consider indecomposable Cartan matrices since the generalization of our results to arbitrary ones is immediate. Also, when the base field has characteristic $P$ different from zero, we always assume, without further mention, that inax $\left\{\left|A_{i j}\right| \mid 1 \leqslant i, j \leqslant l\right\}<P$.

Let $F$ be a field and $\left(A_{i j}\right)$ an $l \times l$ indecomposable Cartan matrix. Let $X$ be the set of $3 l$ symbols $\left\{e_{i}, h_{i}, f_{i} \mid 1 \leqslant i \leqslant l\right\}$ and let $\mathfrak{F}(X)$ denote the free Lie algebra over $F$ on the set $X$. Letting $\mathcal{G}$ denote the ideal of $\mathfrak{F} \mathcal{L}(X)$ generated by the elements $\left[h_{i}, h_{j}\right],\left[e_{i}, f_{j}\right]-\delta_{i j} h_{i},\left[e_{i}, h_{j}\right]-A_{j i} e_{i},\left[f_{i}, h_{j}\right]+A_{j i} f_{i}$ for $1 \leqslant i$, $j<l$, we let $\mathcal{L}$ denote the corresponding factor algebra. $\mathcal{L}$ is called the universal Heffalump algebra over $F$ associated to $\left(A_{i j}\right)$, and if $x \in X$ we again denote its image in $\mathcal{L}$ by $x$.

The following facts about $\mathcal{E}$ are well known and can be found in [1], [4], [5], and [8]. Let $\mathcal{L}^{+}$(respectively $\mathcal{L}^{-}$) be the subalgebra of $\mathcal{L}$ generated by $e_{i}$ (respectively $f_{i}$ ) for $1 \leqslant i \leqslant l$, and let $\mathcal{H}$ be the linear span of $h_{1}, \ldots, h_{l}$. Then $\mathcal{L}=\mathcal{L}^{+} \oplus \mathscr{H} \oplus \mathcal{L}^{-}$and the dimension of the space spanned by $\left\{e_{i}, h_{i}\right.$, $\left.f_{i} \mid 1<i<l\right\}$ is $3 l$. $\mathcal{L}^{+}$(respectively $\mathcal{L}^{-}$) is a free Lie algebra on the set $\left\{e_{i} \mid 1 \leqslant i \leqslant l\right\}$ (respectively $\left\{f_{i} \mid 1 \leqslant i \leqslant l\right\}$ ) and there is an automorphism $\eta$ of period 2 on $\mathcal{E}$ such that $\eta\left(e_{i}\right)=f_{i}, \eta\left(f_{i}\right)=e_{i}, \eta\left(h_{i}\right)=-h_{i}, 1 \leqslant i \leqslant l$. Moreover, if $\left(A_{i j}\right)$ and $\left(B_{i j}\right)$ are equivalent Cartan matrices then the universal Heffalump algebras associated with them are isomorphic. There is an ideal $R$ of $\mathcal{L}$ (see [1], [7]) which is called the radical of $\mathcal{L}$ and, in most cases, $R$ is the unique maximal ideal of $\mathcal{L}$. The corresponding factor algebra is called the reduced Heffalump algebra attached to $\left(A_{i j}\right)$ over $F$.

$\mathcal{L}$ is a graded algebra whose group of degrees, $V_{\mathbf{Z}}$, is a free abelian group of rank $l$ with generators $\alpha_{i}, 1 \leqslant i \leqslant l$. Thus, $\mathcal{L}=\bigoplus_{\alpha \in V_{\mathbf{z}}} \mathcal{L}_{\alpha}, \mathcal{H}=\mathfrak{L}_{0},\left[\mathfrak{L}_{\alpha}, \mathcal{L}_{\beta}\right]$ $\subseteq \mathcal{L}_{\alpha+\beta}$ for all $\alpha, \beta \in V_{\mathrm{Z}}$, and if $e_{\alpha} \in \mathcal{L}_{\alpha}, h \in \mathcal{H}$ then $\left[e_{\alpha}, h\right]=\alpha(h) e_{\alpha}$ where the action of $V_{\mathrm{Z}}$ on $\mathcal{H}$ is determined by $\alpha_{i}\left(h_{j}\right)=A_{j i}$ for $1 \leqslant i, j \leqslant l$. Let $\Gamma=\left\{\alpha \in V_{\mathbf{Z}} \mid \alpha \neq 0, \mathcal{L}_{\alpha} \neq(0)\right\}, \Gamma^{+}=\left\{\alpha \in \Gamma \mid \alpha=\sum_{i=1}^{l} d_{i} \alpha_{i}\right.$ where $d_{i} \geqslant 0$ 
for all $i\}$ and $\Gamma^{-}=-\Gamma^{+}$. Then $\Gamma=\Gamma^{+} \cup \Gamma^{-}$and $\mathcal{L}^{+}=\bigoplus_{\alpha \in \Gamma^{+}} \mathcal{L}_{\alpha}, \mathcal{L}^{-}=$ $\bigoplus_{\alpha \in \Gamma^{-}} \mathcal{L}_{\alpha}$.

If $\alpha=\sum_{i=1}^{l} d_{i} \alpha_{i}$ is a nonzero element of $V_{\mathbf{Z}}$ and $d_{i} \geqslant 0$ for all $i$ then $\alpha \in \Gamma^{+}$ unless $\alpha=n \alpha_{i}$ for some $n \geqslant 2$, because $\mathfrak{L}^{+}$is a free Lie algebra. For $\alpha \in \Gamma$ (or $\alpha \in \mathcal{H}^{*}$ ) we let $[\alpha]=\{\beta \in \Gamma \mid \alpha(h)=\beta(h)$ for all $h \in \mathcal{H}\}$ and let $\mathcal{L}_{[\alpha]}=\bigoplus_{\beta \in[\alpha]} \mathcal{L}_{\beta}$. When our Cartan matrix is not of Euclidean type (see [2] for an enumeration of these) and the characteristic of $F$ is zero we have that the dimension of $\mathcal{L}_{\left[\alpha_{i}\right]}, \mathcal{L}_{\left\{-\alpha_{i}\right]}$ is 1 for $1 \leqslant i \leqslant l$. If $\alpha=\sum_{i=1}^{l} d_{i} \alpha_{i} \in \Gamma$ we let $l(\alpha)=\sum_{i=1}^{l} d_{i}$. Thus, $\mathcal{E}=\bigoplus_{t=-\infty}^{\infty} \mathcal{L}_{t}$ where $\mathcal{L}_{t}=\bigoplus_{\alpha \in \Gamma ; l(\alpha)=t} \mathcal{L}_{\alpha}$. We let $P^{+}$ (respectively $P^{-}$, respectively $P^{0}$ ) denote the projection of $\mathcal{L}$ onto $\mathcal{L}^{+}$ (respectively $\mathcal{L}^{-}$, respectively $\mathscr{H}$ ).

REMARK. The following fact, which is proved using a Hall basis, is of central importance to us. If $x$ and $y$ are two commuting homogeneous elements in a free Lie algebra $\mathfrak{F} \mathcal{E}$, they are linearly dependent [see [6, Theorem 5.10, p. 328]]. We adapt a version of this to our situation in the following result.

LEMMA 1. Let $x, y \in \mathcal{L}$ and assume that $[x, y] \in \mathcal{H}$.

(i) If $P^{+}(x) \neq 0$ then $a P^{+}(x)=P^{+}(y)$ for some $a \in F$.

(ii) If $P^{-}(x) \neq 0$ then $a P^{-}(x)=P^{-}(y)$ for some $a \in F$.

Proof. We only prove (i) since (ii) is similar. Assume $P^{+}(x) \neq 0$ and write $x=\sum_{t=m}^{n} x_{t}, y=\sum_{t=m^{\prime}}^{n^{\prime}} y_{t}$ where $x_{t}, y_{t} \in \mathcal{L}_{t}$, and we assume $n^{\prime} \geqslant 1$ since otherwise the result is trivial. Since $[x, y] \in \mathcal{H}=\mathfrak{L}_{0}$ we have for $k \neq 0$ that $\sum_{s+t=k}\left[x_{t}, y_{s}\right]=0$ where $m^{\prime} \leqslant s \leqslant n^{\prime}, m \leqslant t \leqslant n$. Thus, taking $k=n+n^{\prime}$ yields $\left[x_{n}, y_{n^{\prime}}\right]=0$ so that, by the remark $n=n^{\prime}$ and $a x_{n}=y_{n}$ for some $a \in F$. If $n=1$ we are done, so we assume $n \geqslant 2$ and work by induction. Thus, assume there is some $j$ such that $2 \leqslant j \leqslant n$ and $a x_{t}=y_{t}$ for $j \leqslant t \leqslant n$. Taking $k=n+j-1$ (so that $n+1 \leqslant k \leqslant 2 n-1$ ) we have $0=\left[x_{n}, y_{j-1}\right]$ $+\left[x_{n-1}, y_{j}\right]+\cdots+\left[x_{j}, y_{n-1}\right]+\left[x_{j-1}, y_{n}\right]=\left[x_{n}, y_{j-1}\right]+\left[x_{n-1}, a x_{j}\right]+\cdots$ $\left[x_{j}, a x_{n-1}\right]+\left[x_{j-1}, a x_{n}\right]$. Clearly, all terms cancel except the first and last so we have $0=\left[x_{n}, y_{j-1}\right]+\left[x_{j-1}, a x_{n}\right]=\left[x_{n}, y_{j-1}-a x_{j-1}\right]$. Again using the remark, and noting that $j-1 \neq n$, we get that $y_{j-1}=a x_{j-1}$ as desired. Q.E.D.

We now fix some notation. Let $\left(A_{i j}^{\prime}\right)$ and $\left(A_{i j}\right)$ be two indecomposable Cartan matrices of size $l^{\prime} \times l^{\prime}$ and $l \times l$ respectively, and let $\mathcal{L}^{\prime}$ (respectively $\mathcal{L})$ denote their corresponding universal Heffalump algebras over the field $F$. We use primed and unprimed notation for $\mathcal{L}^{\prime}$ and $\mathcal{L}$ respectively. We assume $l$ and $l^{\prime}$ are not 1 since in that case the results are well known (then the algebra is simple of type $A_{1}$ ). Let $\phi$ denote an isomorphism of $\mathcal{L}^{\prime}$ onto $\mathcal{L}$. We begin our analysis of $\phi$ with the following result.

Lemma 2. There are two nonzero elements $X^{+} \in \mathfrak{L}^{+}, X^{-} \in \mathfrak{L}^{-}$and two linear functionals $\mathfrak{F}^{+}, \mathfrak{F}^{-}$on $\mathcal{H}^{\prime}$ such that, for all $h^{\prime} \in \mathcal{H}^{\prime}, \phi\left(h^{\prime}\right)=\mathfrak{F}^{+}\left(h^{\prime}\right) X^{+}$ $+P^{0}\left(\phi\left(h^{\prime}\right)\right)+\mathfrak{F}^{-}\left(h^{\prime}\right) X^{-}$. 
Proof. If $P^{+}(\phi(\mathcal{H}))=(0)$ we let $X^{+}=e_{1}, F^{+}=0$. Assume next, that $h_{0}^{\prime} \in \mathcal{H}^{\prime}$ is chosen so that $P^{+}\left(\phi\left(h_{0}^{\prime}\right)\right) \neq 0$. Let $X^{+}=P^{+}\left(\phi\left(h_{0}^{\prime}\right)\right)$. Then $X^{+} \neq$ 0 and for any $h^{\prime} \in \mathcal{H}^{\prime}$ we have $\left[\phi\left(h_{0}^{\prime}\right), \phi\left(h^{\prime}\right)\right]=0 \in \mathcal{H}$. By Lemma 1 there is a scalar $\mathfrak{F}^{+}\left(h^{\prime}\right) \in F$ such that $P^{+}\left(\phi\left(h^{\prime}\right)\right)=\mathfrak{F}^{+}\left(h^{\prime}\right) X^{+}$. Similar remarks apply to yield $\mathfrak{F}^{-}$and $X^{-}$, and the result follows. Q.E.D.

The next result is our version of conjugacy for the universal Heffalump algebras.

THEOREM 3. $\phi\left(\mathcal{H}^{\prime}\right)=\mathcal{H}$ so that $l=l^{\prime}$.

Proof. In the notation of Lemma 2 we need only show that $\mathfrak{F}^{+}$and $\mathfrak{F}^{-}$ are zero. For $0 \neq x \in \mathcal{L}$ we write $x=\sum_{t=l^{+}(x)}^{l^{+}(x)}$ where $x_{t} \in \mathcal{L}_{t}, l^{-}(x) \leqslant$ $l^{+}(x)$, and $x_{t} \neq 0$ if either $t=l^{-}(x)$ or if $t=l^{+}(x)$. Because $\phi$ is surjective there is some $\alpha^{\prime} \in \Gamma^{\prime}$ and $e_{\alpha^{\prime}} \in \mathcal{L}_{\alpha^{\prime}}$ such that $l^{+}\left(\phi\left(e_{\alpha^{\prime}}\right)\right)>l^{+}\left(X^{+}\right)$. Say $\phi\left(e_{\alpha^{\prime}}\right)=\Sigma_{\beta \in \Gamma} e_{\beta}$ where $e_{\beta} \in \mathcal{L}_{\beta}$ for all $\beta \in \Gamma$. Then for all $h^{\prime} \in \mathcal{H}^{\prime}$ we have $\left[e_{\alpha^{\prime}}, h^{\prime}\right]=\alpha^{\prime}\left(h^{\prime}\right) e_{\alpha^{\prime}}$. Applying $\phi$ to this yields

$$
\sum_{\beta \in \Gamma} \alpha^{\prime}\left(h^{\prime}\right) e_{\beta}=\sum_{\beta \in \Gamma}\left[e_{\beta}, \mathfrak{F}^{-}\left(h^{\prime}\right) X^{-}+P^{0}\left(\phi\left(h^{\prime}\right)\right)+\mathfrak{F}^{+}\left(h^{\prime}\right) X^{+}\right]
$$

Thus,

$$
\sum_{\beta \in \Gamma}\left(\alpha^{\prime}\left(h^{\prime}\right)-\beta\left(P^{0}\left(\phi\left(h^{\prime}\right)\right)\right)\right) e_{\beta}=\sum_{\beta \in \Gamma}\left(\mathfrak{F}^{-}\left(h^{\prime}\right)\left[e_{\beta}, X^{-}\right]+\mathfrak{F}^{+}\left(h^{\prime}\right)\left[e_{\beta}, X^{+}\right]\right) .
$$

Since $l^{+}\left(\phi\left(e_{\alpha^{\prime}}\right)\right)>l^{+}\left(X^{+}\right)$, the right-hand side of the last equation has a term of degree greater than the left-hand side if $\mathfrak{F}^{+} \neq 0$. Thus, $\mathfrak{F}^{+}=0$ and in a similar way $\mathfrak{F}^{-}=0$. Q.E.D.

REMARK. It follows from the foregoing proof, that if $e_{\alpha^{\prime}} \in \mathcal{L}_{\alpha^{\prime}}$ and $\phi\left(e_{\alpha^{\prime}}\right)=$ $\sum_{\beta \in \Gamma} e_{\beta}$ then $e_{\beta}=0$ unless $\alpha^{\prime}\left(h^{\prime}\right)=\beta\left(\phi\left(h^{\prime}\right)\right)$ for all $h^{\prime} \in \mathcal{H}^{\prime}$. Thus, $\phi\left(\mathcal{L}_{\alpha^{\prime}}\right) \subseteq$ $\mathcal{L}_{[\beta]}$ where $\beta \in \Gamma$ is any root such that $\alpha^{\prime}\left(h^{\prime}\right)=\beta\left(\phi\left(h^{\prime}\right)\right)$ for all $h^{\prime} \in \mathcal{H}^{\prime}$.

2. Classification and automorphism groups. Before going on with our analysis of $\phi$ we need to describe a group of automorphisms acting on $\mathcal{L}$. If $a_{1}, \ldots, a_{l}$ are nonzero scalars from $F$ we let $u\left(a_{1}, \ldots, a_{l}\right)$ be the automorphism of $\mathcal{E}$ such that $e_{i} \rightarrow a_{i} e_{i}, f_{i} \rightarrow a_{i}^{-1} f_{i}$, and $h_{i} \rightarrow h_{i}$. Clearly the inverse of $u\left(a_{1}, \ldots, a_{l}\right)$ is $u\left(a_{1}^{-1}, \ldots, a_{l}^{-1}\right)$. If $\pi$ is a permutation of $\{1, \ldots, l\}$ such that $A_{\pi(i) \pi(j)}=A_{i j}$ for $1 \leqslant i, j \leqslant l$ we let $v(\pi)$ denote the automorphism of $\mathcal{L}$ whose action is determined by $e_{i} \rightarrow e_{\pi(i)}, f_{i} \rightarrow f_{\pi(i)}, h_{i} \rightarrow h_{\pi(i)}$ for $1 \leqslant i \leqslant l$. We let $G(\mathcal{E})$ denote the subgroup of $\operatorname{Aut}(\mathcal{L})$ generated by all these automorphisms together with $\eta$. One easily checks that

$$
u\left(a_{1}, \ldots, a_{l}\right) \eta=\eta u\left(a_{1}^{-1}, \ldots, a_{l}^{-1}\right), \quad v(\pi) \eta=\eta v(\pi),
$$

and that

$$
u\left(a_{1}, \ldots, a_{l}\right) v(\pi)=v(\pi) u\left(a_{\pi(1)}, \ldots, a_{\pi(l)}\right) .
$$

Thus, if $\mathfrak{F} \in G(\mathfrak{L})$ then $\mathfrak{F}$ has the form $v(\pi) u\left(a_{1}, \ldots, a_{l}\right) \eta^{\varepsilon}$ where $\varepsilon \in$ $\{0,1\}$.

We now continue our analysis of $\phi$. The remark following Theorem 3 
implies that $P^{0}\left(\phi\left(e_{i}^{\prime}\right)\right)=0=P^{0}\left(\phi\left(f_{i}^{\prime}\right)\right)$ for $1 \leqslant i \leqslant l$. Thus $\phi\left(e_{i}^{\prime}\right)=E_{i}^{-}+$ $E_{i}^{+}, \phi\left(f_{i}^{\prime}\right)=F_{i}^{-}+F_{i}^{+}$, where $E_{i}^{+}, F_{i}^{+} \in \mathcal{L}^{+}, E_{i}^{-}, F_{i}^{-} \in \mathcal{L}^{-}$for $1 \leqslant i \leqslant l$. Adjusting $\phi$ by $\eta$, if necessary, we assume that $E_{1}^{+} \neq 0$. Then from $\delta_{i j} \phi\left(h_{i}^{\prime}\right)=$ $\left[\phi\left(e_{i}^{\prime}\right), \phi\left(f_{j}^{\prime}\right)\right]$ we get $\delta_{i j} \phi\left(h_{i}^{\prime}\right)=\left[E_{i}^{+},{F_{j}}^{+}\right]+\left[E_{i}^{+},{F_{j}^{-}}^{-}\right]+\left[E_{i}^{-},{F_{j}^{+}}^{+}\right]+\left[E_{i}^{-}\right.$, $F_{j}^{-}$]. Taking $i=1$ and using Lemma 1 , we obtain $F_{j}^{+}=a_{j} E_{1}^{+}$for some $a_{j} \in F, 1 \leqslant j \leqslant l$. If $E_{1}^{-} \neq 0$ we get, in the same way, $F_{j}^{-}=b_{j} E_{1}^{-}$for some $b_{j} \in F, 1 \leqslant j \leqslant l$. Then $\left[E_{1}^{+}, E_{1}^{-}\right] \in \mathcal{H}$, and, letting $\mathcal{S}=F E_{1}^{+}+F E_{1}^{-}+$ $\mathcal{H}$, we have $\phi\left(f_{j}^{\prime}\right) \in \mathcal{S}$ for $1 \leqslant j \leqslant l$, so since $\delta$ is a subalgebra we have $\phi\left(\mathfrak{L}^{\prime-}\right) \subseteq \mathcal{S}$, which is clearly impossible. Thus, $E_{1}^{-}=0$, and hence $F_{1}^{-} \neq 0$ so again by Lemma 1 we have $E_{j}^{-}=C_{j} F_{1}^{-}$for some $C_{j} \in F, 1 \leqslant j \leqslant l$. $C_{1}=0$ and, by symmetry, we have $a_{1}=0$. Also, $\left[E_{1}^{+}, F_{1}^{-}\right] \in \mathcal{H}$.

We now have that $\delta_{i j} \phi\left(h_{i}^{\prime}\right)=\left[E_{i}^{+}, a_{j} E_{1}^{+}\right]+\left[E_{i}^{+}, F_{j}^{-}\right]+\left[C_{i} F_{1}^{-}, a_{j} E_{1}^{+}\right]+$ [ $C_{i} F_{1}^{-}, F_{j}^{-}$]. If some $a_{j} \neq 0$ we appeal to Lemma 1 to get $E_{i}^{+}=d_{i} E_{1}^{+}$for some $d_{i} \in F, 1 \leqslant i \leqslant l$. Then, letting $g$ denote the subalgebra $F F_{1}^{-}+F E_{1}^{+}$ $+\mathcal{K}$, we have $\phi\left(e_{i}^{\prime}\right) \in \mathcal{G}$ for $1 \leqslant i \leqslant l$, hence $\phi\left(\mathcal{L}^{\prime+}\right) \subseteq \mathcal{G}$, and this is impossible. Thus, $a_{j}=0$, and in a similar way $C_{j}=0$, for $1 \leqslant j \leqslant l$.

It now follows that $\Phi\left(\mathcal{L}^{\prime+}\right)=\mathfrak{L}^{+}, \phi\left(\mathcal{L}^{\prime-}\right)=\mathfrak{L}^{-}$. Write $\phi\left(e_{i}^{\prime}\right)=E_{i, 1}+E_{i}^{+}$, where $E_{i, 1} \in \mathfrak{L}_{1}, E_{i}^{+} \in \bigoplus_{t=2}^{\infty} \mathscr{L}_{t}, 1 \leqslant i \leqslant l$.

Since $\phi$ is surjective the dimension of the linear span of $E_{1,1}, \ldots, E_{l, 1}$ is $l$, so that $E_{i, 1} \neq 0$ for $1 \leqslant i \leqslant l$. Also, $E_{i, 1}$ belongs to some root space $\mathfrak{L}_{\alpha}$, and hence there is a permutation $\pi$ of $\{1, \ldots, l\}$ and nonzero scalars $\gamma_{\pi(i)} \in F$ such that $E_{i, 1}=\gamma_{\pi(i)} e_{\pi(i)}$ for $1 \leqslant i \leqslant l$. Similar remarks apply to yield a permutation $\delta$ of $\{1, \ldots, l\}$ and nonzero scalars $\rho_{\delta(i)} \in F$ such that $\phi\left(f_{i}^{\prime}\right)=\rho_{\delta(i)} f_{\delta(i)}+F_{i}^{-}$where $F_{i}^{-} \in \bigoplus_{t=-\infty}^{-2} \mathcal{L}_{t}$ for $1 \leqslant i \leqslant l$.

It is now easy to see that $E_{i}^{+}$is $f$-extreme (i.e. $\left[E_{i}^{+}, f_{j}\right]=0$ for $1 \leqslant j \leqslant l$ ), $F_{i}^{-}$is $e$-extreme, and that $\delta=\pi$. We next adjust $\phi$ by an automorphism of the form $u\left(a_{1}, \ldots, a_{l}\right)$ so that $\phi$ now has the form $\phi\left(e_{i}^{\prime}\right)=e_{\pi(i)}+E_{i}^{+}$, $\phi\left(f_{i}^{\prime}\right)=\rho_{\pi(i)} f_{\pi(i)}+F_{i}^{-}$where $\pi$ is a permutation of $\{1, \ldots, l\}, E_{i}^{+}$is $f$ extreme, $F_{i}^{-}$is $e$-extreme and $0 \neq \rho_{\pi(i)} \in F$ for $1 \leqslant i \leqslant l$. Then $\phi\left(h_{i}^{\prime}\right)=$ $\rho_{\pi(i)} h_{\pi(i)},\left[E_{i}^{+}, h\right]=\alpha_{\pi(i)}(h) E_{i}^{+}$, and $\left[F_{i}^{-}, h\right]=-\alpha_{\pi(i)}(h) F_{i}^{-}$for all $h \in \mathcal{K}$, $1 \leqslant i \leqslant l$. Applying $\phi$ to the relation $\left[f_{i}^{\prime}, h_{j}^{\prime}\right]=-A_{j i}^{\prime} f_{i}^{\prime}$ yields that $\rho_{i}=1$ and $A_{i j}^{\prime}=A_{\pi(i), \pi(j)}$ for $1 \leqslant i, j \leqslant l$. This establishes our main result.

THEOREM 4. Let $\mathcal{L}$ and $\mathcal{L}^{\prime}$ be the universal Heffalump algebras over the field $F$ arising from the indecomposable Cartan matrices $\left(A_{i j}\right)$ and $\left(A_{i j}^{\prime}\right)$ respectively. Then $\mathcal{L}$ is isomorphic to $\mathcal{L}^{\prime}$ if and only if $\left(A_{i j}\right)$ is equivalent to $\left(A_{i j}^{\prime}\right)$.

When the base field is of characteristic 0 and our Cartan matrix is not of Euclidean type we know the dimension of both $\varrho_{\left[\alpha_{i}\right]}$ and $\varrho_{\left\{-\alpha_{i}\right]}$ is 1 for $1 \leqslant i \leqslant l$. From this it follows that any automorphism maps root spaces to root spaces, and this clearly yields the following result.

TheOREM 5. Let $\mathcal{L}$ be a universal Heffalump algebra over a field of characteristic 0 arising from an $l \times l$ indecomposable Cartan matrix. Assume 
$l>2$ and that the Cartan matrix is not of Euclidean type. Then Aut( $(\mathcal{L})$

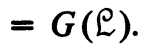

\section{REFERENCES}

1. S. Berman, On the construction of simple Lie algebras, J. Algebra 27 (1973), 158-183. MR 50 \#7270.

2. S. Berman, R. Moody and M. Wonenburger, Cartan matrices with null roots and finite Cartan matrices, Indiana Univ. Math. J. 21 (1971/72), 1091-1099. MR 45 \#3439.

3. S. Berman, On derivations of Lie algebras, Canad. J. Math. 28 (1976), 174-180.

4. N. Jacobson, Lie algebras, Interscience, New York, 1962. MR 26 \# 1345.

5. V. G. Kac, Simple irreducible graded Lie algebras of finite growth, Izv. Akad. Nauk SSSR Ser. Mat. 32 (1968), 1323-1367 = Math. USSR Izv. 2 (1968), 1271-1311. MR 41 \#4590.

6. W. Magnus, A. Karrass and D. Solitar, Combinatorial group theory, Interscience, New York, 1966. MR 34 \#7617.

7. R. Marcuson, Representations and radicals of a class of infinite dimensional Lie algebras, Indiana Univ. Math. J. 23 (1973/74), 883-887. MR 49 \#2863.

8. R. Moody, A new class of Lie algebras, J. Algebra 10 (1968), 211-230. MR 37 \#5261.

9. R. Moody and K. Teo, Tits' systems with crystallographic Weyl groups, J. Algebra 21 (1972), 178-190. MR 47 \#8705.

10. R. Moody, Macdonald identities and Euclidean Lie algebras, Proc. Amer. Math. Soc. 48 (1975), 43-52.

Department of Mathematics, University of Saskatchewan, Saskatoon, Saskatchewan STN OWO, CANADA 